\title{
Use of bone anchors for the treatment of traumatic elbow luxation in dogs: a retrospective case series of three dogs \\ Vasileia Logothetou ${ }^{1,2}$, Eirini Pappa ${ }^{2}$, Rob Pettitt ${ }^{2}$, Eithne J. Comerford ${ }^{2}$ \\ 1. Southfields Veterinary Specialists, Basildon, UK \\ 2. Small Animal Teaching Hospital, School of Veterinary Science, University of Liverpool, Neston, Wirral, UK
}

\section{Introduction}

Traumatic elbow luxation is one of the most common joint luxations in the dog. 1 Surgical treatment options include collateral ligament repair, replacement or reattachment, elastic transarticular external skeletal fixator and circumferential suture repair, or combination of the above. ${ }^{2,3}$ This is the first reported case series on the use of bone anchors for stabilisation of the canine elbow following reduction for a traumatic joint luxation.

\section{Materials and Methods}

Ethical approval was obtained by the ethics panel of the University of Liverpool (UoL). The clinical database of the Small Animal Teaching Hospital of UoL was retrospectively reviewed for cases with traumatic elbow luxation reduced and stabilised with bone anchors between August 2005 and May 2019.

\section{Results}

Three cases with full clinical records were included in the study.

Animal details: All cases presented following a road traffic accident. Cases 1 and 2 had a complete right elbow luxation and case 3 had a left radial head subluxation.

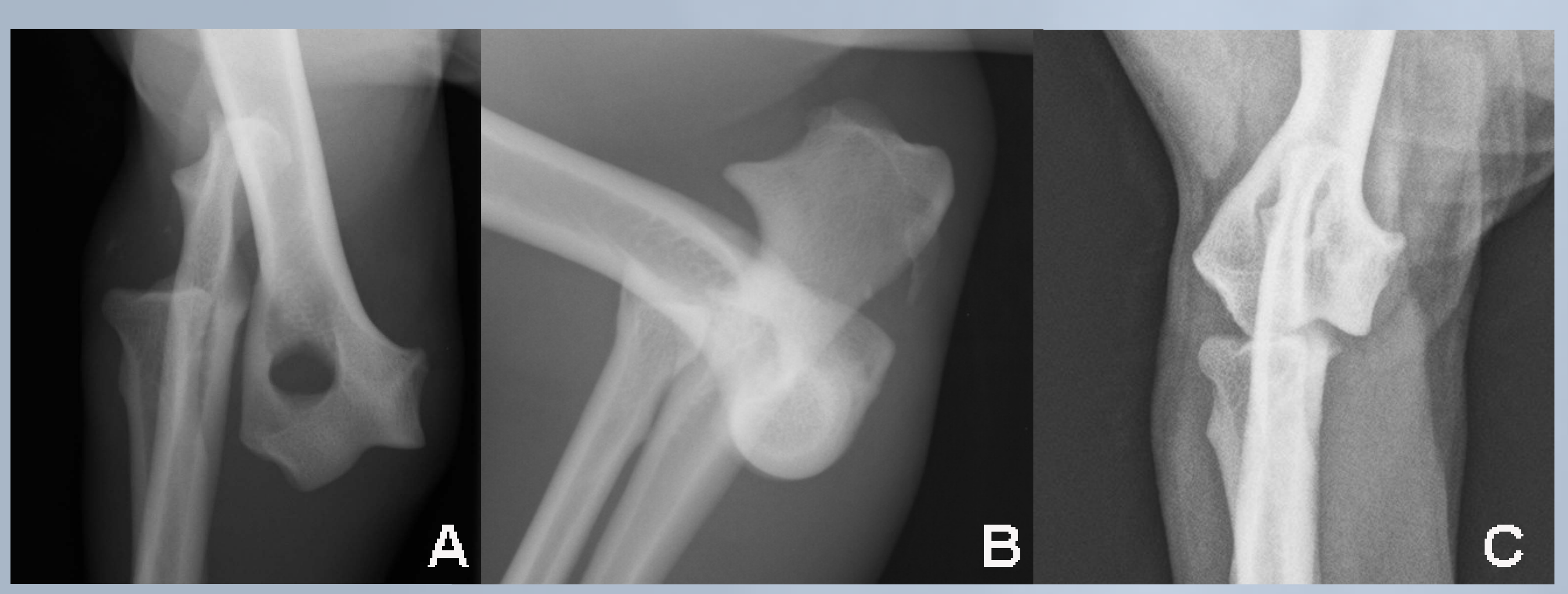
Figure 1: Craniocaudal and mediolateral radiographs of a right lateral elbow
luxation in case $1(A, B)$. Craniocaudal radiograph of the radial head subluxation on case $3(C)$.

Treatment: The elbow joint was approached through a lateral incision in all cases, and the antebrachial fascia was incised. The anconeus muscle was elevated and the tendon of the ulnaris lateralis was transected. The common digital and lateral extensor muscles were elevated to expose the lateral collateral ligament. Cases 1 and 2 had fully torn lateral collateral ligaments. Case 3 had a frayed lateral collateral ligament. All elbows were reduced and stabilised with a bone anchor on the lateral condyle. Case 1 and 2 had IMEX $4 \mathrm{~mm}$ bone anchors implanted, with polydioxanone $2-0$. Case 3 had a FASTak ${ }^{\circledR}$ $2.4 \mathrm{~mm}$ anchor implanted with 2-0 FiberWire. A locking loop pattern was used to attach the suture to the distal collateral ligament.
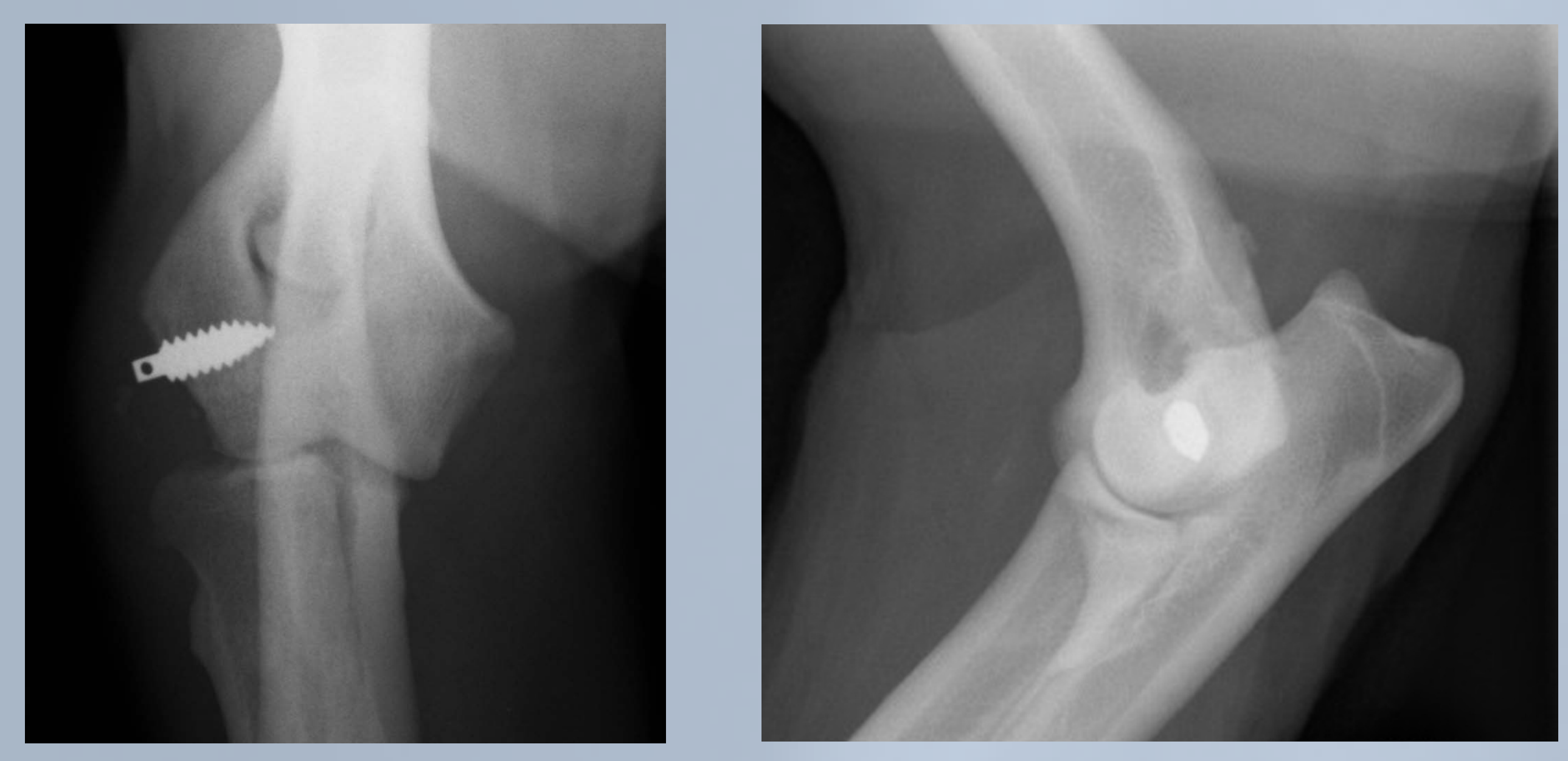

Figure 2: Craniocaudal and mediolateral postoperative radiographs in case 2 after stabilisation of the right elbow with an IMEX ${ }^{\text {TM }}$ bone anchor.
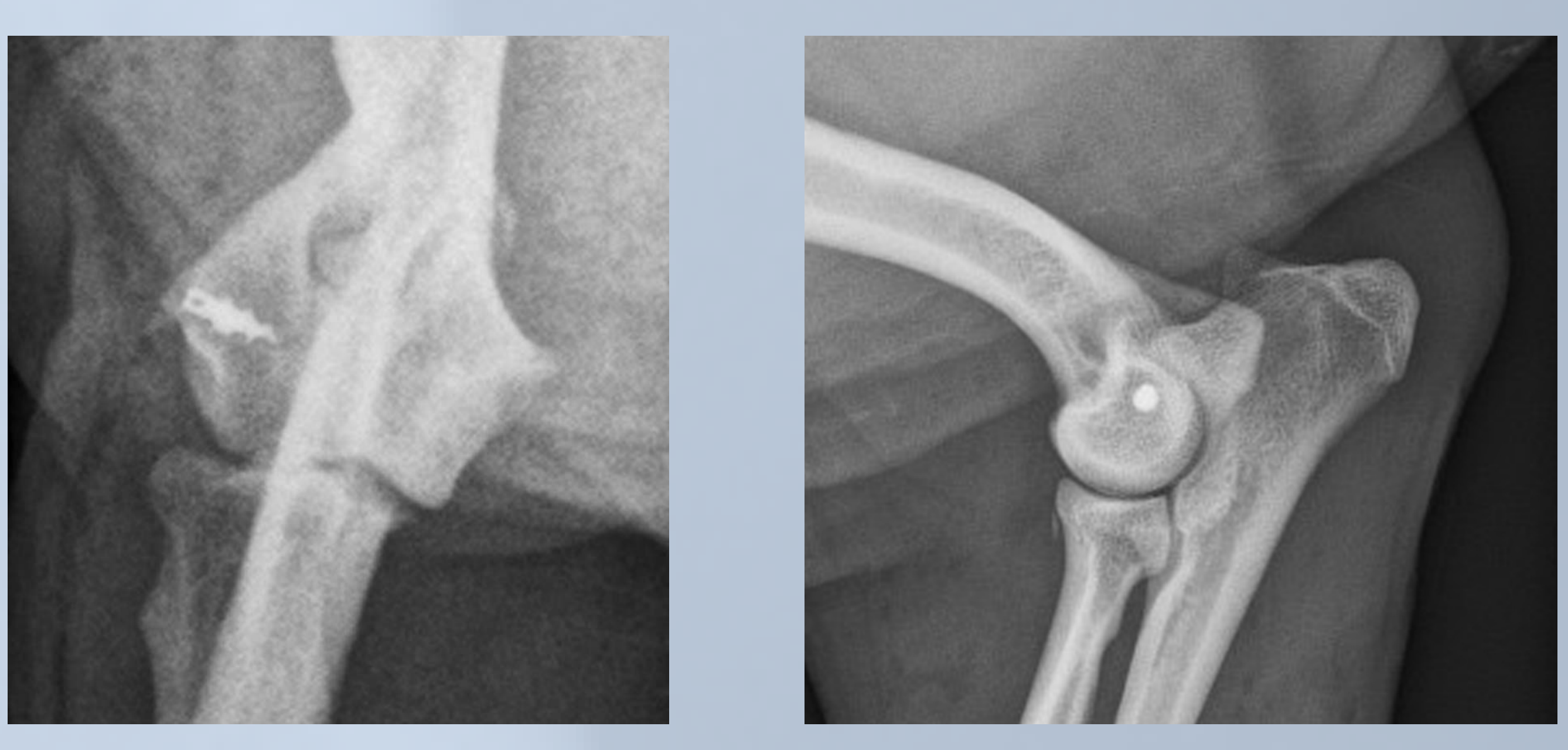

Figure 3: Craniocaudal and mediolateral postoperative radiographs of the left elbow in case 3 after stabilisation with a FASTak ${ }^{\circledR}$ bone anchor.

Post-operative care: Only one case had a bandage placed immediately postoperatively.

Complications: Case 1 had a reluxation 24 hours postoperatively. Reduction and stabilisation was performed with the same technique and application of a spica splint. A nonabsorbable suture (polypropylene 1) was placed through the bone anchor and sutured with Bunnell suture pattern to the distal part of the ruptured collateral ligament.

Follow up: Radiographic evaluation revealed good elbow congruency in 2 and 7 weeks after surgery in cases 2 and 3. Long term follow up was available in cases 1 and 3 , with 8 years and 6 months respectively. Normal function of the affected limb was noted. Case 1 had occasional mild episodes of lameness which did not require long-term medication. A Liverpool Osteoarthritis Questionnaire (LOAD) was completed by the owners of case 3. A LOAD score of zero was obtained, indicating normal gait perception by the owners prior to the injury and 24 weeks postoperatively.
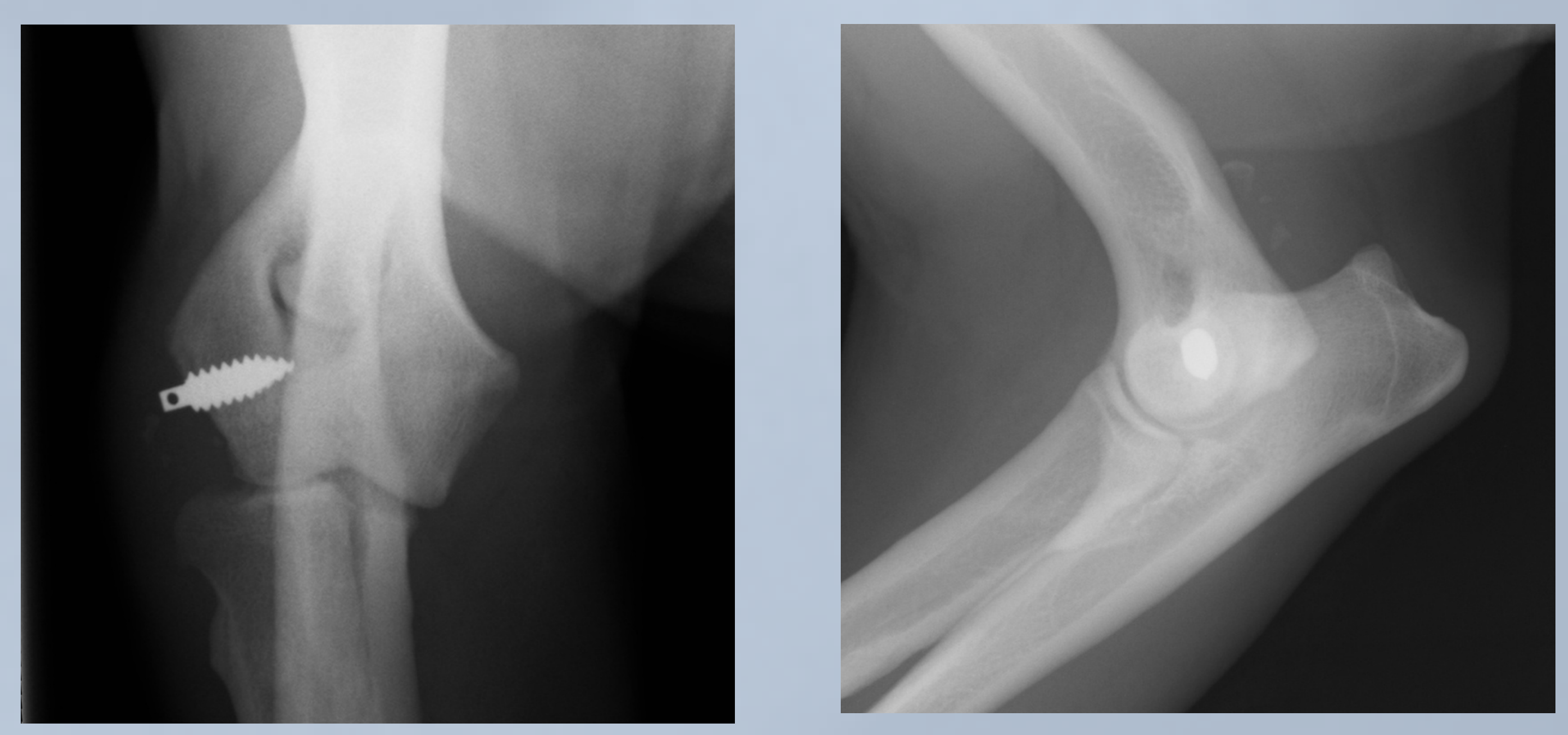

Figure 4: Craniocaudal and mediolateral radiographs of the right elbow 7 weeks postoperatively, in case 2 . Minimal osteoarthritic changes are visible.

\section{Discussion}

Historically, closed reduction was recommended as the treatment of choice for traumatic elbow luxations. 1,4,5 In more recent studies, surgical treatment options have increased popularity. ${ }^{2}$ Compared to other techniques, bone anchors offer a single incision approach, with no necessary additional equipment and reduced complications. The bone anchors offer a robust attachment for augmentation of the damaged or ruptured ligament. We describe an easy approach and technique that has excellent short term and long term outcomes, with high owner satisfaction.

\section{References}

Campbell JR. Luxation and Ligamentous Injuries of the Elbow of the Dog. Veterinary Clinics of North America. 1971;1(3):429-40.

Sajik D, Meeson RL, Kulendra N, Jordan CJ, James D, Calvo I, et al. Multi-centre retrospective study of long-term outcomes following traumatic elbow luxation in 37 dogs. J Small Anim Pract. 2016;57(8):422-8.

Bordelon JT, Reaugh HF, Rochat MC. Traumatic luxations of the appendicular skeleton. Vet Clin North Am Small Anim Pract. 2005;35(5):1169-94.

O'Brien MB, Boudrieau RJ, Clark GN. Traumatic luxation of the cubital joint (elbow) in dogs: 44 cases (1978-1988). Journal of the American Veterinary Medical Association. 1992;201 11:1760-5.

Mitchell KE. Traumatic elbow luxation in 14 dogs and 11 cats. Aust Vet J. 2011;89(6):213- 